\title{
Heat Transfer Design for Bionic Surfaces in a Simplified Transition Segment of Marine Gas Turbine Combustor
}

\author{
Long MA, Tao XU, Tianyi ZHANG, Zhenglei YU, Haotian GUO
}

\begin{abstract}
Gas turbine is a widely-used propulsion device for power convection in marine dynamical system. Conventional coolants such as impingement cooling and thermal protection material have lower convective heat transfer efficiency on the target surface which can hinder the development of marine gas turbine combustor. In this paper, impact cooling simplification models are established, which have simulated the function of the transition segment of marine gas turbine combustor to be protected from high temperature. Being enlightened by the butterfly scale, four types of bionic ribs are designed on the simplification models. During the analysis, conservation equations for mass, momentum and energy are solved by using finite volume method with Realizable $k-\varepsilon$ turbulence model. By comparing the four types bionic ribs models, the surface with Type 3 rib structure has the best cooling efficiency. The results show that the sharp corner and unequal length fins of bionic rib could affect the cooling efficiency. The inspire application of the bionic structures will provide a reference for new cooling structure design in marine gas turbine combustor.
\end{abstract}

Keywords: bionic thermal surface; computational fluid dynamics (CFD); convective heat transfer; marine gas turbine

\section{INTRODUCTION}

Gas turbine is a power machinery which is widely used in ships, especially warships, because of its light weight per unit power, compact size, high speed, high flexibility and fast start-up time [1]. However, the gas turbine's thermal efficiency is low owing to the fact that it exhausts highgrade heat to the atmosphere. In order to improve the thermal efficiency of the gas turbine, the inlet temperature need to increase, which depends on advancements in material and cooling technology $[2,3]$. The researchers found that improved heat resistance of material cannot meet the requirement of the thermal protection of gas turbine components, both external and internal cooling strategies are supposed to be used to dissipate heat from the gas turbine effectively [4].

In the past few decades, many researchers had conducted in-depth studies in cooling methods, impingement cooling and rib/groove turbulated cooling were typical methods for external and internal cooling methods, respectively. For internal cooling method, a comprehensive experimental had been carried out on ribbed ducts [5-7]. Xie [8] designed six cases with different ribbed channels to study the influence of the rectangular ribs arrangement. All cases were simulated with $v^{2}-f$ model, ribs with different sizes were staggered and the distance between ribs was various in different cases. The results exhibited that the downstream ribs play an important role in enhancing heat transfer at high Reynolds number range, and certain arrangements had distinct influence on their thermal enhancement factor. Using the Reynolds-averaged Navier-Stokes (RANS) and $k-\omega$ SST turbulence model, Saha and Prabhudharwadkar [9] studied the hemispherical groove, teardrop groove and teardrop groove-angled broken groove which were applied to gas turbine blade. Through the comparative analysis of the averaged Nusselt number values $N u / N u_{0}$, teardrop shaped groove presented better heat transfer performance than other groove geometries. In addition, all the geometries had almost the same friction factor ratio $f / f_{0}$, which means that three kinds of grooves produce the same range of pressure drop. With the development of bionics, some researchers applied creature micro-structures to surface. Cui [10] simplified four typical non-smooth surfaces (inspired by the sharks' skin, the jaegers' wings, the seals' shin and the shells' stripes, respectively). According to the inspirations, placoid-shaped grooves, V-shaped grooves, riblet-shaped grooves and ridge-shaped grooves were studied, respectively. The simulation results revealed that ridgeshaped grooved-surface had the best performance in drag reduction.

Similarly, natural creatures can provide inspiration for cooling methods of the gas turbine. Butterfly is widely present in the nature of the Lepidoptera animals, their wings generally show a rich colour. It has been found that most of the colours of the butterfly wings are caused by a special flake structure on the wings. Butterfly scale structure is shown in Fig. 1 which likes tower-shaped [11]. The nanostructures have the effect of interference, scattering on the incident light, so that the light incident on the surface of the scale is reflected or scattered back at a large angle. The different colours are seen at different angles [12].

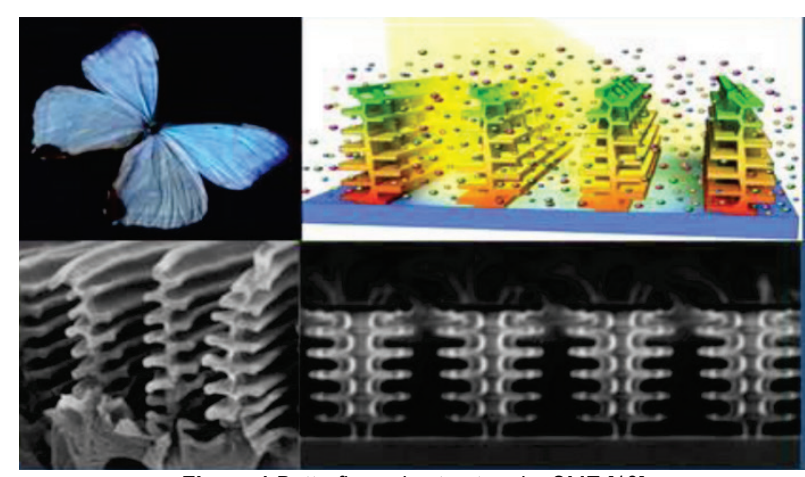

Figure 1 Butterfly scale structure by SME [13]

In the natural world, the sun radiates the energy by the radiation of the electromagnetic wave. Since the heat rays and the optical characteristics are the same, the rules of projection, reflection and refraction of light are also applicable to the heat rays. Koon [14] found that the butterfly through the scales of micro-nano surface to capture the sun, thus forming a 'super black' surface. By using electron microscopy and transition electron microscopy, $\mathrm{Wu}[15]$ analyzed the butterfly scale structure, 
simulation of its structure to create biomimetic materials to promote solar thermal utilization. Scientists had found that the butterflies regulate the amplitude of sunlight by opening and closing scales, thus having a self-regulating body temperature function. These studies have surface butterfly flake structures that have an effect on heat radiation.

As the connection structure, the transition segment of the marine gas turbine combustor connects the combustion chamber flare tube and turbine, and the temperature of the combustion products is $1800 \mathrm{~K}$ to $2000 \mathrm{~K}$. This seriously restricts the ship maneuverability. So it is necessary to consider the cooling of the high-temperature components. In the work presented here, a bionic surface which is inspired from the butterfly scale structure is established to improve the surface heat transfer capacity, and consider the radiation factor, analyses the effect of butterfly scale rib on surface heat transfer. The bionic structure has a large effective surface heat transfer area and a better influence on the radiation ability. The results presented in this paper can be used as a reference for future experimental verification and technical implementation of practical marine gas turbine applications.

\section{METHOD OF MUMERICAL ANALYSIS \\ 2.1 Physical Model}

There are about 1400 kinds of butterflies all over the world. Among the different kinds of butterflies, the tropical Morpho butterfly is the most widely studied for its special optical behaviour.

Table 1 Parameters of four types of bionic ribs $(\mathrm{mm})$

\begin{tabular}{|c|c|c|c|c|c|c|c|c|}
\hline Type & Fin1. $L$ & Fin2. $L$ & Fin3. $L$ & Fin4. $L$ & Fin5. $L$ & $t$ & $s$ & $\begin{array}{c}\text { If has } \\
\text { fillet }\end{array}$ \\
\hline 1 & 3.2 & 3.2 & 3.2 & 3.2 & 3.2 & 1.6 & 1.6 & No \\
\hline 2 & 3.2 & 3.2 & 3.2 & 3.2 & 3.2 & 1.6 & 1.6 & Yes \\
\hline 3 & 1.0 & 1.55 & 2.1 & 2.65 & 3.2 & 1.6 & 1.6 & No \\
\hline 4 & 1.0 & 1.55 & 2.1 & 2.65 & 3.2 & 1.6 & 1.6 & Yes \\
\hline
\end{tabular}

Inspired by the photonic nanoarchitecture of Morpho butterfly scales in Tab. 1, this paper designs four types of bionic rib applied to the high-temperature surface of the simplified rectangular marine gas turbine transition segment. The detailed parameter and sectional shape are depicted in Fig. 2 and described as follows.

Type 1: the side with three pins faces which have the same length and without fillet.

Type 2: the size is the same as the type 1, except for the fins that are with fillet.

Type 3: the side with three pins faces which have different lengths and are without fillet.

Type 4: the size is the same as the type 3, except for the fins that are with fillet.

The new transition piece (TP) of marine gas turbine combustor features a rounded body shape that balances the heat transfer loading both internally and externally and eliminates resonant frequency concerns, which consists of heavier walls, single-piece aft ends, ribs, seal arrangements, and selective cooling. It has an upstream aperture for the gas flow and it is used to receive the gas flow directly from the corresponding combustion liners with a high level of enthalpy; they are conjured in a longitudinal direction so that their downstream ends comprise arched segments to form a ring-type configuration which opens toward the first stage of the gas turbine.
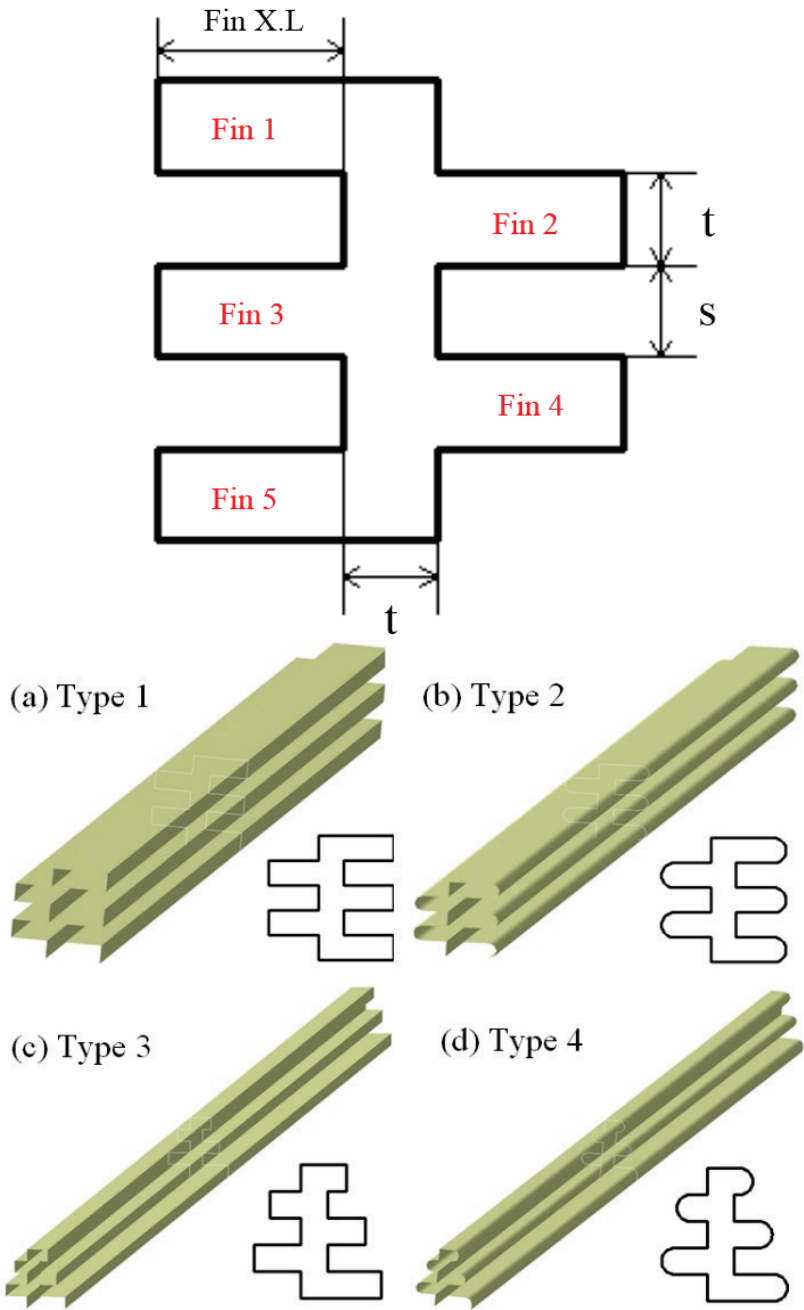

Figure 2 Sectional shape of bionic ribs

The discrete coolant jets, forming a protective film chamber on the side of transition piece, are drawn from the upstream compressor in an operational gas turbine engine. The coolant flows fed through internal passages with surface holes. From the supply plenum, the coolants ejected through several rows of discrete-holes over the external boundary layer against the local high thermal conduction on the other side of the TP.

A simplified double chamber model is established in this work. A schematic of the flow domain along with boundary conditions and dimensions is given in Fig. 3. By using the bionic structure, the surfaces could be highlighted by the ribs spoiler structure on the impact of the strengthening effect of shock. The inner surface of the bionic spoiler model, taking into account the convenience of processing, can use rectangular approximation ribs to achieve the simulation of the impact of cooling process. The size of the model is: axial $(X)$ length $1050 \mathrm{~mm}$, internal gas chamber height of $162 \mathrm{~mm}$, heat transfer surface thickness of $6.8 \mathrm{~mm}$, cooling medium cavity height of 38 $\mathrm{mm}$, diversion lining wall thickness of $3.2 \mathrm{~mm}$. The diversion lined with three cold air inlets, the center of model is located at $400 \mathrm{~mm}$ from the outer wall (at the 
negative end of the $X$-axis), distributed radially on the baffled surface and $10.26 \mathrm{~mm}$ in diameter.

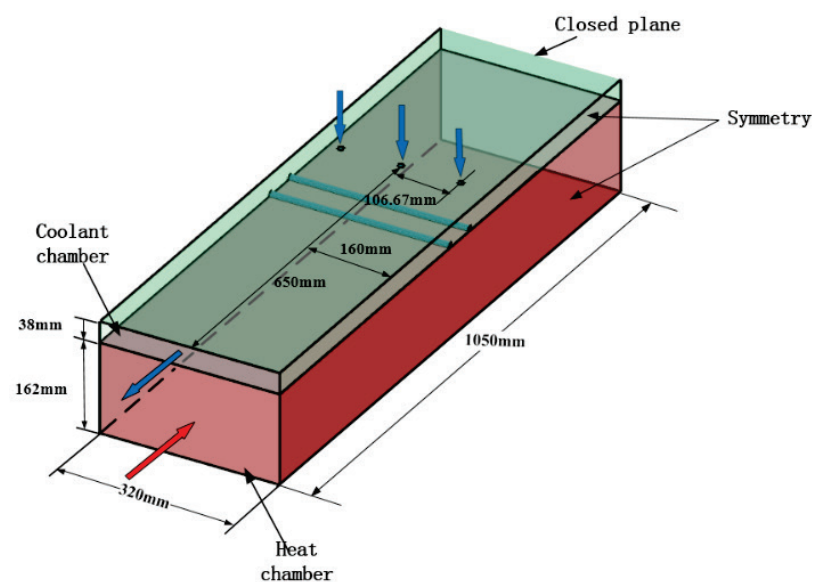

Figure 3 The structure of simplified rectangular marine gas turbine transition segment

\subsection{Governing Equations}

In order to simplify the computation models in the precondition of the comparative results and select a suitable model for CFD simulation, some assumptions are considered as:

(1) Nativer-Stokes and energy equations are applicable;

(2) Steady, incompressible flows;

(3) Gravitational ambient and buoyancy force is neglected;

(4) DTRM model is used to simulate radiative heat transfer;

(5) Realizable $k-\varepsilon$ model is used to simulate the turbulent flow and heat transfer.

Continuity equation [16]:

$\operatorname{div}(U)=\frac{\partial u}{\partial x}+\frac{\partial v}{\partial y}+\frac{\partial w}{\partial z}=0$

In Eq. (1): $u$ - the velocity vectors in $x$ directions, $\mathrm{m} / \mathrm{s} ; v$ the velocity vectors in $y$ directions, $\mathrm{m} / \mathrm{s} ; w$ - the velocity vectors in $z$ directions, $\mathrm{m} / \mathrm{s}$.

Momentum equation:

$\frac{\partial \rho u_{j} u_{i}}{\partial x_{j}}=-\frac{\partial p}{\partial x_{i}}+\frac{\partial}{\partial x_{j}}\left[\left(\mu+\mu_{t}\right)\left(\frac{\partial u_{j}}{\partial x_{i}}+\frac{\partial u_{i}}{\partial x_{j}}\right)\right]$

Energy equation:

$\frac{\partial u_{j} T}{\partial x_{j}}=\frac{\partial}{\partial x_{j}}\left[\left(\frac{\mu}{\operatorname{Pr}}+\frac{\mu_{t}}{\operatorname{Pr}_{t}}\right) \frac{\partial T}{\partial x_{i}}\right]$

Stevens - Boltzmann's Law:

$E_{b}=\sigma T^{4}=C_{0}\left(\frac{T}{100}\right)^{4}$
In Eq. (4): $\sigma$ - blackbody radiation constant, $5.67 \times 10^{-8}$ $\mathrm{W} /\left(\mathrm{m}^{2} \cdot \mathrm{K}^{4}\right) ; C_{0}$ - blackbody radiation coefficient and $b$ represents blackbody, $5.67 \mathrm{~W} /\left(\mathrm{m}^{2} \cdot \mathrm{K}^{4}\right)$.

\subsection{Mesh and Numerical Simulation}

The commercial software CATIA is used to build the simplified geometric model of the transition segment of the gas turbine combustor. ICEM CFD is used to divide the grid by orthogonal hexahedron meshes. There is a large gradient in normal velocity near the boundary layer; the grid of the area directly affects the calculation accuracy. One method normally applied is using the dimensionless value known as $y^{+}$, which is used to calculate the height of the first layer grid.

$\operatorname{Re}=\frac{\rho u L}{\mu}$

In Eq. (5): Re - the Reynolds number; $\rho$ - the fluid density; $u$ - the flow velocity; $L$ - the feature length; $\mu$ - the dynamic viscosity.

$C_{f}=0.058 \cdot \mathrm{Re}^{-0.2}$

In Eq. (6): $C_{f}$ - the wall friction coefficient.

$\tau_{w}=\frac{1}{2} C_{f} \rho u^{2}$

In Eq. (7): $\tau_{w}$ - the wall shear stress.

$U_{\tau}=\sqrt{\frac{\tau_{w}}{\rho}}$

In Eq. (8): $U_{\tau}$ - the friction velocity.

$y=\frac{y^{+} \mu}{U_{\tau} \rho}$

In Eq. (9): $y$ - the wall distance.

Fig. 4 shows the grid information about the whole computational domain. The meshes are generated as HEXA type by ICEM/CFD. The grid systems of mainstream and the cooling chambers are both H-type. For the accuracy and validity of the numerical results, the grid sensitivity has been checked carefully. Four sets of mesh specifications have been adopted. The refinement of a mesh with about 2.5 times of the initial cells was chosen for the analysis. In Tab. 2, the calculated results of the transition segment of the gas turbine combustor with smooth surface are given. Different mesh specifications yield similar results. The differences of the weighted average temperature are smaller than 5\%. The mesh density does not change the weighted average temperature in any appreciable way. The size of the mesh is 0.05 . The total numbers of the cells for the four models are $2,252,456,2,369,027,2,044,248,2,146,701$. For the four models, all nodes on the inner wall surface have the $y$ plus vale smaller than 200 . 
Table 2 Validity of the grid number for air weighted average temperature

\begin{tabular}{|c|c|c|}
\hline Case & $\begin{array}{c}\text { Global max element seed } \\
\text { size }(\mathrm{mm})\end{array}$ & $\begin{array}{c}\text { Weighted average } \\
\text { temperature }(\mathrm{K})\end{array}$ \\
\hline 1 & 10 & 1230 \\
\hline 2 & 8 & 1204 \\
\hline 3 & 6 & 1182 \\
\hline 4 & 4 & 1180 \\
\hline
\end{tabular}

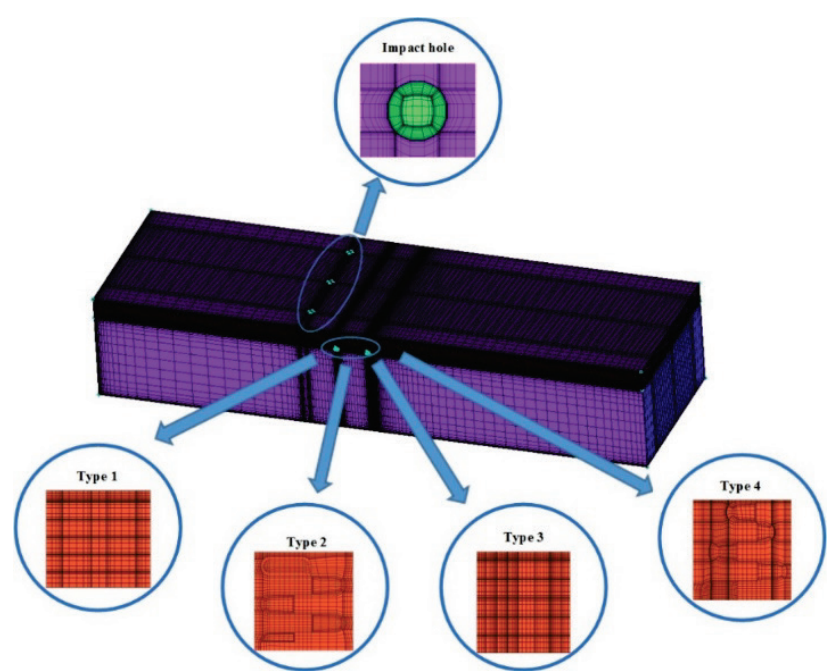

Figure 1 The mesh image of the main flow field

\subsection{Boundary Conditions}

The top surface and the mid surface are made of nickel and Nickel 263 respectively, their thermo physic characters are given in Tab. 3 [5].

Table 3 Boundary conditions of the simulation

\begin{tabular}{|c|l|c|}
\hline \multirow{2}{*}{ Position } & \multicolumn{1}{|c|}{ Boundary condition } & value \\
\hline \multirow{4}{*}{ Mainstream inlet } & Mass flux rate & $32.72 \mathrm{~kg} / \mathrm{s}$ \\
\cline { 2 - 3 } & Gas temperature & $1300 \mathrm{~K}$ \\
\cline { 2 - 3 } & Turbulent intensity & $5 \%$ \\
\cline { 2 - 3 } Mainstream outlet & Hydraulic diameter & $0.324 \mathrm{~m}$ \\
\hline \multirow{3}{*}{ Coolant chamber } & Turbulent intensity & $5 \%$ \\
\cline { 2 - 3 } & Convection coefficient & $10 \mathrm{~W} /\left(\mathrm{m}^{2} \cdot \mathrm{K}\right)$ \\
\cline { 2 - 3 } & Hydraulic diameter & $0.324 \mathrm{~m}$ \\
\hline & Air temperature & $300 \mathrm{~K}$ \\
\cline { 2 - 3 } & Turbulent intensity & $10 \%$ \\
\cline { 2 - 3 } & Hydraulic diameter & $0.01026 \mathrm{~m}$ \\
\hline
\end{tabular}

\section{RESULTS AND DISCUSSION}

\subsection{Parameter Definition}

To understand the coolant flow behaviour and convective heat transfer characteristics in the rectangular channels, some equations are defined as below.

In order to intuitively acquire the quantity of heat that is taken away in the process of heat exchange in the cooling chamber, the flow rate temperature $\lambda$, which provided as an indicator is computed by the product of flow rate and defined.

$\lambda=\int T \rho \vec{v} \cdot \mathrm{d} \vec{A}=\sum_{i=1}^{n} T_{i} \rho_{i} \vec{v}_{i} \cdot \vec{A}_{i}$

In Eq. (10): $\rho$ - the density of the coolant; $\vec{v}$ - the facet velocity on the selected field.

The heat transfer coefficient can be defined as $\eta=\frac{\beta_{i}-\beta_{s}}{\beta_{s}}$

where it serves as an indicator to value the performance of impinging cooling. In Eq. (11): $i$ - the number of group; $s$ the group of smooth thermal surface.

Where $\beta$ is defined as

$\beta=\lambda_{\text {out }}-\lambda_{\text {in }}$

The friction factor $f$ is introduced by:

$f=\frac{\Delta p}{2 \rho u_{i}^{2}} \cdot \frac{D_{h}}{L}$

In Eq. (13): $\Delta p$ - the pressure difference of drops from inlet to outlet; $L$ - the length of overall channel; $D_{h}$ - the diameter of the channel; $u_{i}$ - the velocity of the flow.

\subsection{Comparison of Cooling Efficiency}

To explore the effect of different shapes of rib structure on cooling efficiency of the transitional double chamber model, some numerical simulation results related to temperature are shown in Tab. 4 and Fig. 5. The temperature distribution on thermal surface is shown in Fig. 6.

Table 4 Flow rate temperature and cooling efficiency for different ribs on therma surface

\begin{tabular}{|c|c|c|c|c|c|}
\hline Case & Type & $\lambda_{\text {in }}(\mathrm{K} \cdot \mathrm{kg} / \mathrm{s})$ & $\lambda_{\text {out }}(\mathrm{K} \cdot \mathrm{kg} / \mathrm{s})$ & $T_{v}(\mathrm{~K})$ & $\eta(\%)$ \\
\hline 1 & Smooth & & -83.61 & 1180.72 & 12.00 \\
\hline 2 & Square rib & 49.67 & -112.00 & 1146.63 & 15.34 \\
\hline 3 & Type1 rib & 50.36 & -127.88 & 1094.64 & 20.54 \\
\hline 4 & Type2 rib & 50.42 & -116.20 & 1112.30 & 18.77 \\
\hline 5 & Type3 rib & 50.38 & -131.22 & 1089.23 & 21.08 \\
\hline 6 & Type4 rib & 50.65 & -125.25 & 1105.81 & 19.42 \\
\hline
\end{tabular}



Figure $\mathbf{5}$ The curves of heat transfer coefficient and minimum temperature on the thermal surface

The flow rate temperature of the coolant inlet and outlet is represented by $\lambda_{\text {in }}$ and $\lambda_{\text {out }}$, respectively. $\lambda_{\text {in }}$ floats slightly around $50 \mathrm{~K} \cdot \mathrm{kg} / \mathrm{s}$, which can be considered as a constant value. The value of $\lambda_{\text {out }}$ varies from -83.61 to $-131.22 \mathrm{~K} \cdot \mathrm{kg} / \mathrm{s}$. Positive values indicate inflow cooling direction. The negative values mean coolant flows out of the cooling chamber. Since the calculation results are 
obtained under steady state conditions, the flow rate in outlet is fixed. The $\lambda_{\text {out }}$ with larger absolute value means coolant takes more heat out of the chamber. In all models, the absolute value of $\lambda_{\text {out }}$ is ranked as: Type $3 \mathrm{rib}>$ Type 1 rib>Type 4 rib>Type 2 rib $>$ square rib>smooth, which is the same order as cooling efficiency. The results show that four types of ribs designed in this study inspired on butterfly scale have higher cooling efficiency than the traditional square rib and smooth surface. In detail, Type 3 and 1 ribs without fillet are surrounded by sharp corners, and their cooling efficiency is higher than Type 4 and 2 which have fillet in the fins. In addition, the lengths of fins could also affect the cooling efficiency. In the Type 3 and 4 ribs, their fins' lengths decrease gradually from bottom to top, they exhibit better cooling efficiency than Type 1 and 2, respectively. Therefore, the sharp corner and unequal length fins can improve the cooling performance. Compared with the other types, the Type 3 rib has both features, and has better cooling efficiency than the others have.

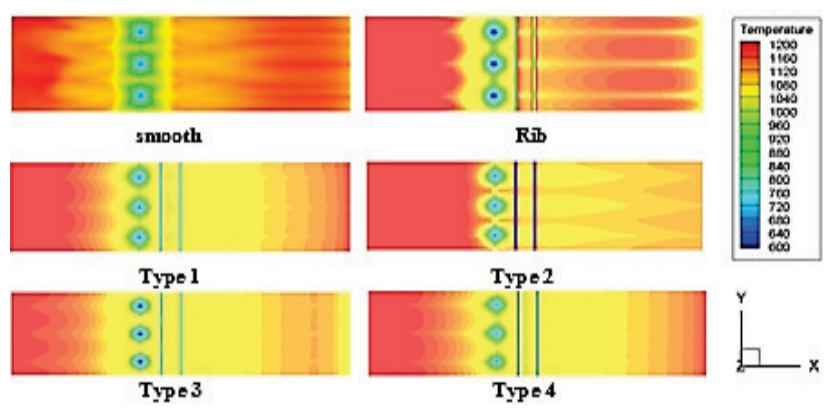

Figure 6 Comparisons of temperature distribution on the thermal surfaces

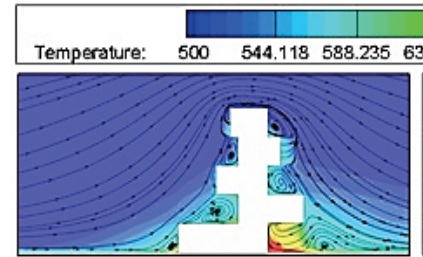

Type 3 (lst row)

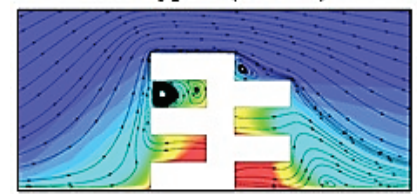

Type 1 (lst row)

Figure 7 The flow streamlines and temperature contours around $1^{\text {st }}$ and $2^{\text {nd }}$ streamwise row of ribs

When the coolant flows through the thermal surface, boundary layer will be formed in the contact facets with the wall surface. The velocity of the coolant flow near the wall surface is almost zero. The heat transfer is mainly conducted by heat conduction. The thermal conductivity of the coolant is very low, which makes the heat transfer efficiency reduced. The formation of the boundary layer is not conducive to the improvement of the convective heat transfer on the wall surface. Bionic ribs can obstruct and change the flow direction of the streamline, destroy the boundary layer and increase the cooling capacity. In order to visualize how ribs influence temperature distribution by changing the flow feature. Fig. 7 shows the streamlines and temperature contours around the ribs at stream wise- normal planes. The direction of the arrow in the figure represents the flow direction, the $1^{\text {st }}$ row represents the rib near the impact hole, and the $2^{\text {nd }}$ row represents the rib far from the impact hole. Near the ribs at the impingement hole, the velocity of the fluid is large, and there are three fins with sharp corners in the upwind direction. Each of the acute angles affects the direction of movement of a part of the fluid. When the fluid receives a sharp angle, there is room above it to make it small. The small swirling structure accelerates the heat exchange between the coolant and the rib surface.

\section{CONCLUSIONS}

In this study, four types of bionic ribs are designed based on the wings of butterflies, used on the thermal surface of the transitional double chamber model. Through the simulation of the marine gas turbine working condition, the heat transfer of the biomimetic thermal surface has been investigated by CFD. The main conclusions drawn from the study are concluded as follows:

(1) Compared with the smooth model, the surface with bionic ribs structure gives better cooling efficiency than the smooth model at the same conditions which could improve jet impingement destabilization.

(2) The bionic ribs with sharp corner and unequal length fins could improve the cooling performance. By comparing the four type models, the cooling efficiency of Type 3 is $21.08 \%$, which has both two features and best cooling performance.

It is significant to consider the effect of heat conduction within the metal on the predictions of an accurate surface temperature and hence impingement cooling effectiveness. In the future, the structure parameters of the bionic ribs could be optimized, such as length, width, height and other ribs' size parameters, number and shape of the fins, the distance between the ribs, et al, which could affect the cooling efficiency directly. We expect this work will be employed in designing heat component of Marine Gas Turbine Combustor.

\section{Acknowledgements}

The authors would like to acknowledge the National Key R\&D Program of China (2018YFB1105100), National Natural Science Funds for Distinguished Young Scholar (Grant No. 51505181), the Advanced Manufacturing Project of Provincial School Construction of Jilin Province (No. SXGJSF2017-2) and the China Postdoctoral Science Foundation funded project (No. 2016M590256)

\section{REFERENCES}

[1] Hou, S. Y., Wu, Y. D., Zhou, Y. D., \& Yu, L. J. (2017). Performance analysis of the combined supercritical $\mathrm{CO}_{2}$ recompression and regenerative cycle used in waste heat recovery of marine gas turbine. Energy Conversion and Management, 151, 73-85. https://doi.org/10.1016/j.enconman.2017.05.042

[2] Chandrasekaran, N. \& Guha, A. (2013). Development and optimization of a sustainable turbofan aeroengine for improved performance and emissions. Proceedings of the 
Institution of Mechanical Engineers, Part G: Journal of Aerospace Engineering, 227(11), 1701-1719. https://doi.org/10.1177/0954410012462183

[3] Han, J. C., Duffa, S., \& Ekkad, S. V. (2013). Gas turbine heat transfer and cooling technology. New York, NY: Yaylor \& Francis.

[4] Zhao, N. B., Wen, X. Y., \& Li, S. Y. (2016). An evaluation of the application of nanofluids in intercooled cycle marine gas turbine intercooler. Journal of Engineering for Gas Turbines and Power, 138, 012201-1-9. https://doi.org/10.1115/1.4031170

[5] Yu, Z. L., Xu, T., Li, J. L., Ma, L., \& Xu, T. S. (2013). Comparison of a series of double chamber model with various hole angles for enhancing cooling effectiveness. International Communications in Heat and Mass Transfer, $44,38-44$. https://doi.org/10.1016/j.icheatmasstransfer.2013.03.002

[6] Wang, X. L., Yan, H. B., Lu, T. J., Song, S. J., \& Kim, T. (2014). Heat transfer characteristics of an inclined impinging jet on a curved surface in crossflow. International Journal of Thermal Sciences, 136, 081702-1-10. https://doi.org/10.1016/j.ijthermalsci.2013.11.005

[7] Won, S. Y. \& Ligrani, P. M. (2004). Comparisons of flow structure and local Nusselt numbers in channels with parallel- and crossed-rib turbulators. International Journal of Heat and Mass Transfer, 47(8-9), 2004, 1573-1586. https://doi.org/10.1016/j.ijheatmasstransfer.2003.10.026

[8] Xie, G. N., Zheng, S. F., Zhang, W. H. \& Sundén, B. (2013) A numerical study of flow structure and heat transfer in a square channel with ribs combined downstream half-size or same-size ribs. Applied Thermal Engineering, 61(2), 289300. https://doi.org/10.1016/j.applthermaleng.2013.07.054

[9] Saha, K. \& Prabhudharwadkar, D. (2015). Performance evaluation of a modified cross section grooved channel as turbulence promoter in internal cooling channel of a gas turbine blade. ASME 2015 International Mechanical Engineering Congress and Exposition. https://doi.org/10.1115/IMECE2015-51428

[10] Cui, J. \& Fu, Y. (2012). A numerical study on pressure drop in microchannel flow with different bionic micro-grooved surfaces. Journal of Bionic Engineering, 9(1), 99-109. https://doi.org/10.1016/S1672-6529(11)60102-9

[11] Lippert, W. \& Gentil, K. (1959). Über lamellare Feinstrukturen bei den Schillerschuppen der Schmetterlinge vom Urania- und Morpho-Typ. Zoomorphology, 48, 115122. https://doi.org/10.1007/BF00407836

[12] Wu, L. Y. (2006). Microstructure and reflective optical properties of typical butterfly scales. Jilin, Changchun: Jilin University.

[13] Han, Z. W., Niu S. C., Shang C. H., Liu Z. N., \& Ren L. Q. (2012). Light trapping structures in wing scales of butterfly Trogonoptera brookiana. Nanoscale, 4, 2879-2883. https://doi.org/10.1039/C2NR12059C

[14] Koon, D. W. \& Crawford, A. B. (2000). Insect thin films as sun blocks, not solar collectors. Applied Optics, 39(15), 2496-2498. https://doi.org/10.1364/AO.39.002496

[15] Wu, L. Y., Qiu, Z. M., \& Song, Y. Q. (2013). Bionic study on structural solar absorption materials based on microstructure pattern of butterfly scales. Advanced Materials Research, 609, 190-194. https://doi.org/10.4028/www.scientific.net/AMR.608-609.190

[16] Goldstein, R. J. \& Behbahani A. I. (1982). Impingement of a circular jet with and without cross flow. International Journal Heat Transfer, 25, 1377-1382. https://doi.org/10.1016/0017-9310(82)90131-4

\section{Contact information:}

\section{Long MA}

School of Mechanical and Aerospace Engineering,

Jilin University \& Theory Department of Aviation, Avation Unversity of Air Force, No. 5988, Renmin Street, Changchun, Jilin Province, 130022, P. R. China

Tao XU

School of Mechanical and Aerospace Engineering, Jilin University, No. 5988, Renmin Street, Changchun, Jilin Province, 130022, P. R. China

\section{Tianyi ZHANG}

School of Mechanical and Aerospace Engineering, Jilin University, No. 5988, Renmin Street, Changchun, Jilin Province, 130022, P. R. China

\section{Zhenglei YU}

(Corresponding author)

Key Lab of Bionic Engineering, Ministry of Education, Jilin University, No. 5988, Renmin Street, Changchun, Jilin Province, 130022, P. R. China yuzhenglei@hotmail.com

\section{Haotian GUO}

School of Mechanical and Aerospace Engineering, Jilin University, No. 5988, Renmin Street, Changchun, Jilin Province, 130022, P. R. China 